\title{
Imatinib-induced fulminant liver failure in chronic myeloid leukemia: role of liver transplant and second-generation tyrosine kinase inhibitors: a case report
}

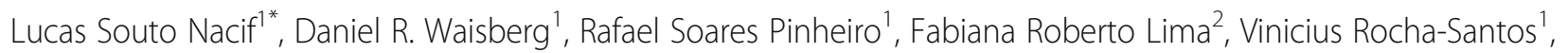
Wellington Andraus ${ }^{1}$ and Luiz Carneiro D'Albuquerque ${ }^{1}$

\begin{abstract}
Background: There is a worldwide problem of acute liver failure and mortality associated with remaining on the waiting for a liver transplant. In this study, we highlight results published in recent years by leading transplant centers in evaluating imatinib-induced acute liver failure in chronic myeloid leukemia and follow-up in liver transplantation.

Case presentation: A 36-year-old brown-skinned woman (mixed Brazilian race) diagnosed 1 year earlier with chronic myeloid leukemia was started after delivery of a baby and continued for 6 months with imatinib mesylate (selective inhibitor of Bcr-Abl tyrosine kinase), which induced liver failure. We conducted a literature review using the PubMed database for articles published through September 2017, and we demonstrate a role of liver transplant in this situation for imatinib-induced liver failure. We report previously published results and a successful liver transplant after acute liver failure due to imatinib-induced in chronic myeloid leukemia treatment.

Conclusions: We report a case of a successful liver transplant after acute liver failure resulting from imatinib-induced chronic myeloid leukemia treatment. The literature reveals the importance of prompt acute liver failure diagnosis and treatment with liver transplant in selected cases.
\end{abstract}

Keywords: Acute liver failure, Liver transplantation, Organ graft, Transplantation

\section{Background}

Liver transplant (LT) is an excellent therapeutic option for acute liver failure (ALF) [1]. Rapid clinical manifestation and severe hepatic injury can arise from many drugs used to treat ALF [1]. However, some situations are very unusual in clinical practice and can be related to oncological blood diseases, including chronic myeloid leukemia (CML).

Imatinib-induced ALF in a patient with CML was first described as being managed with dasatinib [2]. Even though severe hepatotoxicity may be observed in $1-4 \%$ of patients treated with imatinib, cases of fulminant liver

\footnotetext{
*Correspondence: lucasnacif@usp.br

1 Liver and Gastrointestinal Transplant Division, Department of

Gastroenterology, University of São Paulo School of Medicine, Rua Dr. Enéas

de Carvalho Aguiar, 255-9o andar-sala 9113/9114, São Paulo, SP

CEP05403-900, Brazil

Full list of author information is available at the end of the article
}

failure are rare, with only eight known cases [2-9]. All cases but one involved patients with CML, and most of the cases were fatal [3-7]. LT was reported in four of them $[2,5,8,9]$, with one postoperative death [5]. Nilotinib was used after LT in two cases [8, 9], and Harding et al. first described dasatinib therapy [2]. In this report, we review the literature and report another successful case of LT for imatinib-induced liver failure.

\section{Case presentation}

A 36-year-old brown-skinned woman (mixed Brazilian race) was admitted to our institution with jaundice, nausea, mild right upper abdominal pain, choluria, and acholia of 1 week's duration. She had been diagnosed with CML 1 year before (during her 22nd week of pregnancy). Treatment with standard dose (400 mg/day) imatinib (Gleevec; Novartis, Basel, Switzerland) had been started after 
delivery and was continued for 6 months, with no adverse effects until 45 days prior to admission, when a mild elevation in liver enzymes was observed (aspartate transaminase [AST] $229 \mathrm{IU} / \mathrm{L}$, alanine transaminase [ALT] $111 \mathrm{IU} / \mathrm{L}$ ).

Imatinib was discontinued, but the patient presented on admission with ALT $1422 \mathrm{IU} / \mathrm{L}$, AST $1690 \mathrm{IU} / \mathrm{L}$, total bilirubin $13.08 \mathrm{mg} / \mathrm{dl}$, prothrombin time $>50$ (international normalized ratio 2.38), fibrinogen $<50 \mathrm{mg} / \mathrm{dl}$, and factor V Leiden $11 \%$. An abdominal ultrasound revealed no abnormalities. The results of viral screening were negative for hepatitis B (hepatitis B surface antigen, anti-hepatitis B core antibody), hepatitis C (anti-hepatitis $\mathrm{C}$ virus), human immunodeficiency virus (anti-HIV-I/II), cytomegalovirus (immunoglobulin G [IgG]-positive and IgM-negative), and Epstein-Barr virus. The results were also negative for autoimmune hepatitis (antimitochondrial antibody, anti-LKM1 antibody to liver kidney microsome type 1 , and antinuclear antibody). The patient's copper serum levels were normal $(39 \mu \mathrm{g} / \mathrm{dl})$. The laboratory findings associated with hyperammonemia and clinical manifestation of grade II/III encephalopathy led to the diagnosis of ALF.

The patient met the King's College (O'Grady) and Clichy criteria and then underwent urgent orthotopic LT the next day. The graft weighed $0.930 \mathrm{~kg}$. Total ischemic time and warm ischemic time were 6.9 hours and $35 \mathrm{mi}-$ nutes, respectively. The donor was a 40 -year-old woman with brain death resulting from a ruptured brain aneurysm. The donor presented the following extended criteria: vasoactive drug use (noradrenalin $0.05 \mu \mathrm{g} / \mathrm{kg}$ / minute and vasopressin $0.1 \mu \mathrm{g} / \mathrm{kg} /$ minute), infection (nosocomial pneumonia, use of piperacillin/tazobactam for 3 days), and long intensive care unit stay (17 days). The postoperative transaminase peaks were ALT and AST of $668 \mathrm{IU} / \mathrm{L}$ and $760 \mathrm{IU} / \mathrm{L}$, respectively. The immunosuppressive regimen featured steroids (STs) and calcineurin inhibitors as well as tacrolimus (Libbs, São Paulo, Brazil). This regimen was administered as a ST bolus during the anhepatic phase (methylprednisolone $500 \mathrm{mg}$ ) and was decreased to $20 \mathrm{mg} /$ day and tapered to a complete stop within 6 months of LT. Tacrolimus was orally administered and maintained $(0.10-0.15 \mathrm{mg} / \mathrm{kg} /$ day administered orally twice per day every 12 hours).

The patient had a good evolution during the postoperative period and was tracheally extubated on the second day. However, on the fourth postoperative day, she underwent a laparotomy day because of hemodynamic instability, coagulopathy, increased drainage through abdominal drains, and serum hemoglobin decrease after hemotransfusion without major bleeding. On the sixth postoperative day, the patient presented acute dyspnea and pulmonary congestion. Further investigation with pulmonary angiographic computed tomography ruled out pulmonary embolism. An echocardiogram showed an ejection fraction of $35 \%$ (previously 65\%) without ischemic cardiac or coronary abnormalities. These symptoms were attributed to myocardiopathy secondary to systemic inflammatory response syndrome (SIRS). We excluded all other causes of cardiomyopathy (vascular, muscular, or infectious involvement, including sepsis). The patient presented a satisfactory evolution with low doses of diuretics and beta-blockers (carvedilol). She was discharged on the 25th postoperative day.

The histopathological report revealed severe acute hepatitis (Fig. 1a-d) with submassive hepatic necrosis, which is compatible with the patient's subacute hepatic failure. Upon gross examination, the liver weighed $436 \mathrm{~g}$, and the Glisson's capsule was focally wrinkled. Histological examination showed that the more preserved parenchyma had a green to tan appearance in contrast to some irregular reddish-brown zones representing extensive necrotic areas. The left lobe was more severely damaged than the right.

Microscopically, areas of panacinar and multiacinar necrosis were intermixed with large areas of less affected parenchyma exhibiting confluent and bridging necrosis. The inflammatory infiltrate in portal tracts varied in intensity, usually with a predominance of mononuclear cells. Other findings included remarkable ductular reaction, collapsed reticulin framework, significant cholestasis, mild steatosis, hepatic enzyme induction, acidophilic bodies, and regeneration of hepatocytes. Plasma cells were not prominent. Neither granuloma formation nor eosinophil-rich infiltrate was detected. No components or any evidence of CML was found in the liver explant.

The patient remained asymptomatic for CML; nevertheless, her $B C R-A B L$ transcript rose to $148 \% 4$ months after LT. It was $0.41 \%$ prior to imatinib withdrawal. Dasatinib (Bristol-Myers Squibb, New York, NY, USA) was then introduced at $60 \mathrm{mg} /$ day because of the patient's prior history of myocardiopathy related to SIRS. No adverse effects were observed, and the patient's $B C R-A B L$ transcript reduced to $10.64 \%$ after 2 months.

At follow-up, the patient's liver function laboratory tests (total bilirubin, transaminase levels, and coagulation profile), as well as her vascular and biliary tract (ultrasound and magnetic resonance imaging), remained normal 10 months after the LT. The patient returned to her daily routine.

\section{Ethical aspects}

This study was approved by the institutional review board of our institution and fulfilled all of the requirements for studies in humans. It adhered to the guidelines of the 1975 Declaration of Helsinki.

\section{Discussion}

Imatinib mesylate is a selective inhibitor of Bcr-Abl tyrosine kinase and is the first-line treatment for patients 


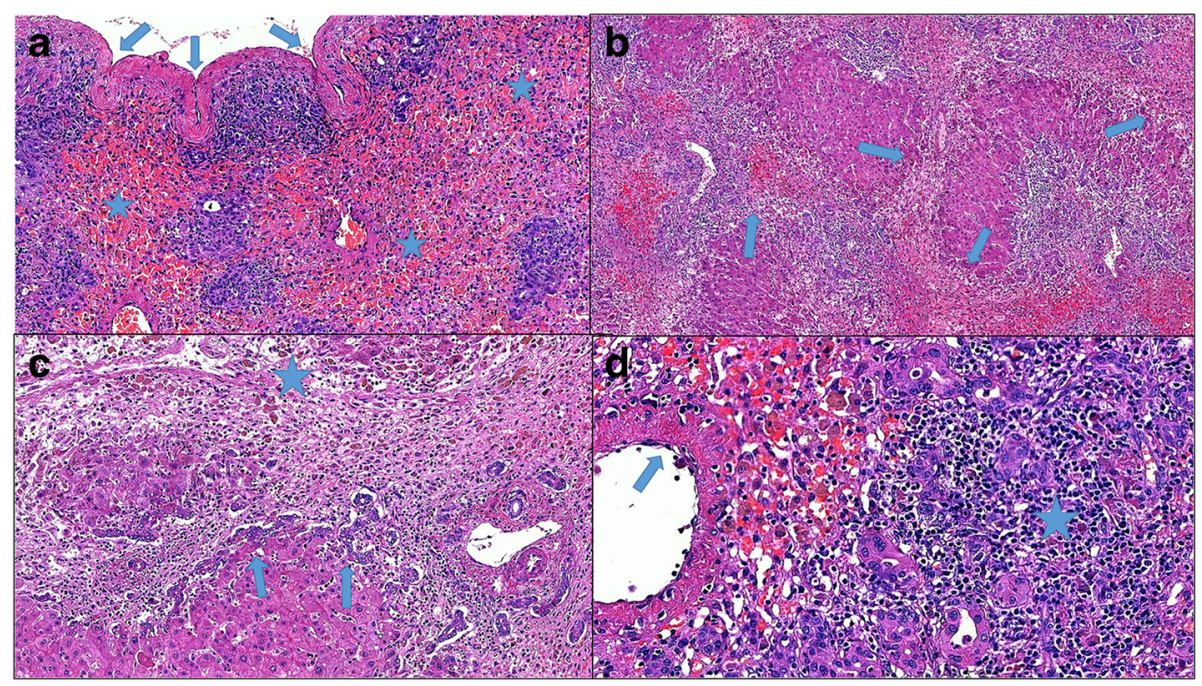

Fig. 1 a A large area with multiacinar necrosis accompanied by hemorrhage and parenchymal collapse (stars). This resulted in liver shrinkage and wrinkling (arrows) of the Glisson's capsule (hematoxylin and eosin stain, original magnification $\times 100$ ). $\mathbf{b}$ Architectural distortion with bridging necrosis (arrows) linking central and portal zones, collapse, and hepatocellular regeneration (hematoxylin and eosin stain, original magnification $\times 50$ ). c Periportal necrosis and central portal bridging (star), prominent ductular reaction (arrows), loose connective tissue stroma with liver cell loss, inflammation, and clusters of pigment-laden macrophages (hematoxylin and eosin stain, original magnification $\times 100$ ). $\mathbf{d}$ This portal tract (on the right side of the image) is expanded by significant mononuclear inflammatory infiltrate (star) and ductular structures (whose cells have a variable morphology including intermediate forms). A central vein shows venulitis (arrow) and is located adjacent to the periportal zone because of panacinar necrosis (hematoxylin and eosin stain, original magnification $\times 200$ )

with CML without a suitable bone marrow donor or in whom transplant would be inadvisable [3]. It is also used for the systemic treatment of advanced gastrointestinal stromal tumors (GISTs) [10]. Hepatic toxicity is usually resolved with either dosage reduction or discontinuation of imatinib [3], which has been permanently required in $0.5 \%$ of patients [6]. In this scenario, second-generation tyrosine kinase inhibitors such as dasatinib, nilotinib, sunitinib, and regorafenib may be used $[2,8,9,11,12]$.

Imatinib-related severe acute hepatitis has been described in 11 cases with favorable outcome after drug interruption (all cases) and use of prednisolone (four cases) [10]. Nine patients were female, two were male, and their age ranged from 22 to 64 years. Nine cases were CML and two were GISTs. It is interesting to note that the time between imatinib start and hepatotoxicity varied from less than 2 weeks to 77 weeks in these cases.

Table 1 presents nine cases of imatinib-related fulminant liver failure found in the literature. No ST treatment was attempted in any of these cases. The time between imatinib start and hepatotoxicity varied from 6 days to 77 weeks; nonetheless, all but two cases had at least 20 weeks of imatinib use. LT was performed in five cases.

The pathogenesis of hepatotoxicity due to imatinib is unclear and may be related to hazards of drug association. Cytochrome P450 enzymes such as CYP3A4, CYP2C9, and CYP2D6 are responsible for imatinib metabolism [5, 10]. Inhibitors of CYP3A4, such as

Table 1 Cases of imatinib-related fulminant liver failure in the literature

\begin{tabular}{|c|c|c|c|c|c|c|}
\hline Author [reference] & Year & Patient age and sex & Diagnosis & $\begin{array}{l}\text { Time between imatinib } \\
\text { start and hepatotoxicity }\end{array}$ & Treatment & Outcome \\
\hline Talpaz et al. [3] & 2002 & Not reported & CML & 6 days & Drug interruption & Death \\
\hline Lin et al. [4] & 2003 & 61 years, female & PV & 7 weeks & Drug interruption & Death \\
\hline Cross et al. [5] & 2006 & 46 years, female & $C M L$ & 77 weeks & LT & Death \\
\hline Ridruejo et al. [6] & 2007 & 51 years, female & CML & 20 weeks & Drug interruption & Death \\
\hline Thia et al. [7] & 2008 & 45 years, female & CML + chronic hepatitis B & 20 weeks & Drug interruption & Death \\
\hline Perini et al. [8] & 2009 & 47 years, female & CML & 72 weeks & LT & Survived \\
\hline Martínez Pascual et al. [9] & 2010 & 34 years, female & $\mathrm{CML}$ & 32 weeks & LT & Survived \\
\hline Harding et al. [2] & 2016 & 30 years, female & CML & 20 weeks & LT & Survived \\
\hline Present case & 2017 & 36 years, female & CML & 30 weeks & LT & Survived \\
\hline
\end{tabular}

Abbreviations: CML chronic myeloid leukemia, PV Polycythemia vera, $L T$ Liver transplant 
erythromycin, clarithromycin, itraconazole, roxithromycin, levonorgestrel, ethinylestradiol, cyproterone (present in some oral contraceptives), and grapefruit juice, increase imatinib concentration and can result in toxicity. In two cases of severe acute hepatitis, patients were receiving acetaminophen as well [10]. Duloxetine is metabolized by CYP1A2 and CYP2D6 pathways. These were present in one patient who ultimately underwent LT [8]. Therefore, caution should be taken when administering other hepatotoxic drugs.

Even though nilotinib and dasatinib are structurally similar to imatinib and are metabolized by the CYP3A4 pathway, previous reports have confirmed a lack of crossintolerance. Its use appears to be safe even in cases of severe toxicity associated with imatinib. However, sunitinib and regorafenib have been associated with fatal fulminant liver failure [11] and severe acute hepatitis [12], respectively. In both cases, patients had advanced GISTs that were unresponsive to imatinib. Interestingly, no previous hepatotoxicity had been observed with this association.

Although rare, ALF in patients with oncological diseases results in ethical dilemmas. There is a scarcity of liver grafts and high mortality of patients on a waitlist. Transplant patients who may have limited survival owing to tumor recurrence might not be the best candidates. They can also suffer more than most from immunosuppression. On the other hand, fulminant liver failure is an emergency that leads to death within a few days unless the patient undergoes LT. Therefore, is it justified to abruptly shorten oncological patients' survival if they have a favorable prognosis? There is no definitive answer. Thus, the decision must be individualized to each patient and deeply discussed by the LT recipient and the oncology teams.

\section{Conclusions}

Imatinib-related CML cases are candidates for LT, given the favorable outcome of the few cases reported in the literature and the possibility of using equally effective medications during the post-transplant period.

\section{Abbreviations}

ALF: Acute liver failure; ALT: Alanine transaminase; AST: Aspartate transaminase; CML: Chronic myeloid leukemia; CYP: Cytochrome P450 enzyme;

GIST: Gastrointestinal stromal tumor; Ig: Immunoglobulin; LT: Liver transplant; PV: Polycythemia vera; SIRS: Systemic inflammatory response syndrome; ST: Steroid

\section{Acknowledgements}

Not applicable.

\section{Funding}

Not applicable.

\section{Availability of data and materials}

All data presented in this manuscript are provided with in-text citations with corresponding references provided in the References section. No datasets were generated or analyzed during this study.

\section{Authors' contributions}

DRW and LSN conceived of and designed the study; collected, analyzed, and interpreted data; and wrote the manuscript. RSP conceived of and designed the study; collected, analyzed, and interpreted data; and critically revised the manuscript. FRL and VRS collected, analyzed, and interpreted data and critically revised the manuscript. WA and LCD'A conceived of the study, interpreted data, and critically revised the manuscript. All authors approved the final manuscript.

Ethics approval and consent to participate

Not applicable.

\section{Consent for publication}

Written informed consent was obtained from the patient for publication of this case report and any accompanying images. A copy of the written consent is available for review by the Editor-in-Chief of this journal.

\section{Competing interests}

The authors declare that they have no competing interests. No writing assistance was used in the production of this manuscript.

\section{Publisher's Note}

Springer Nature remains neutral with regard to jurisdictional claims in published maps and institutional affiliations.

\section{Author details}

${ }^{1}$ Liver and Gastrointestinal Transplant Division, Department of Gastroenterology, University of São Paulo School of Medicine, Rua Dr. Enéas de Carvalho Aguiar, 255-9o andar-sala 9113/9114, São Paulo, SP CEP05403-900, Brazil. '2Department of Pathology, University of São Paulo School of Medicine, São Paulo, Brazil.

Received: 8 December 2017 Accepted: 27 January 2018

Published online: 10 March 2018

\section{References}

1. Hessheimer AJ, Nacif L, Flores Villalba E, Fondevila C. Liver transplantation for acute liver failure. Cir Esp. 2017;95(4):181-9.

2. Harding DJ, MacQuillan G, Howman R, de Boer B, Adams L, Mitchell A, Jeffrey G. Successful use of dasatinib after liver transplantation for imatinibinduced fulminant liver failure in chronic myeloid leukemia. J Clin Oncol. 2016;34(11):e97-8.

3. Talpaz M, Silver R, Druker B, Goldman J, Gambacorti-Paserini C, Guilhot F, et al. Imatinib induces durable hematologic and cytogenetic responses in patients with accelerated phase chronic myeloid leukemia: results of a phase 2 study. Blood. 2002;99:1928-37.

4. Lin N, Sarantopoulos S, Stone J, Gallinsky I, Stone R, Deangelo D, et al. Fatal hepatic necrosis following imatinib mesylate therapy. Blood. 2003;102:3455-6.

5. Cross T, Bagot C, Portmann WJ, Gillet D. Imatinib mesylate as a cause of acute liver failure. Am J Hematol. 2006;81:189-92.

6. Ridruejo E, Cacchione R, Villamil AG, Marciano S, Gadano AC, Mandó OG. Imatinib-induced fatal acute liver failure. World J Gastroenterol. 2007;13:6608-11.

7. Thia TJK, Tan HH, Chuah THC, Chow WC, Lui HF. Imatinib mesylate-related fatal acute hepatic failure in a patient with chronic myeloid leukaemia and chronic hepatitis B infection. Singap Med J. 2008;49:e86-9.

8. Perini G, Santos F, Funke V, Ruiz J, Ferraz B, Hamerschlak N. Nilotinib postliver transplantation for acute hepatic failure related to imatinib. Leuk Res. 2009:33:e234-5.

9. Martínez Pascual C, Valdés Mas M, de la Peña Moral JM, Miras López M. Fulminating hepatitis for imatinib in a patient with chronic myeloid leukaemia [in Spanish]. Med Clin (Barc). 2011;137(7):329-30.

10. Tonyali O, Coskun U, Yildiz R, Karakan T, Demirci U, Akyurek N, Benekli M, Buyukberber S. Imatinib mesylate-induced acute liver failure in a patient with gastrointestinal stromal tumors. Med Oncol. 2010;27(3):768-73.

11. Weise AM, Liu CY, Shields AF. Fatal liver failure in a patient on acetaminophen treated with sunitinib malate and levothyroxine. Ann Pharmacother. 2009:43(4):761-6.

12. Akamine T, Ando K, Oki E, Saeki H, Nakashima Y, Imamura YU, Ohgaki K, Maehara Y. Acute liver failure due to regorafenib may be caused by impaired liver blood flow: a case report. Anticancer Res. 2015;35(7):4037-41. 